Article

\title{
Analysis of Topological Endomorphism of Fuzzy Measure in Hausdorff Distributed Monoid Spaces
}

\author{
Susmit Bagchi \\ Department of Aerospace and Software Engineering (Informatics), Gyeongsang National University, \\ Jinju 660701, Korea; profsbagchi@gmail.com
}

Received: 30 March 2019; Accepted: 11 May 2019; Published: 15 May 2019

\begin{abstract}
The concepts of fuzzy sets and topology are widely applied to model various algebraic structures and computations. The dynamics of fuzzy measures in topological spaces having distributed monoid embeddings is an interesting topic in the presence of topological endomorphism. This paper presents the analysis of topological endomorphism and the properties of topological fuzzy measures in distributed monoid spaces. The topological space is considered to be Hausdorff and second countable in nature. The analysis of consistency of fuzzy measure in endomorphic topological spaces is formulated. The algebraic structures of endomorphic topological spaces having distributed cyclic monoids are constructed. The cyclic monoids contain specific generators, and a related cyclic topological endomorphism within the subspace is formulated. The analytical properties of fuzzy topological measures in the presence of cyclic topological endomorphism are presented. A comparative analysis of this proposed work with other related work in the domain is included.
\end{abstract}

Keywords: Hausdorff; monoid; fuzzy sets; fuzzy measures; topological space; endomorphism

\section{Introduction}

Algebraic groups are often symmetry- and orientation-preserving structures with applications in physics and cryptography. Furthermore, topological structures are widely applied in analyzing geometric shapes and computing spaces. The properties of endomorphism are important in detecting inherent symmetries and uniqueness between spaces. In general, algebraic structures are distinguished from topological structures, and a topological structure embedding a group is called a topological group [1]. Topological groups incorporate uniformity within the underlying algebraic group structure. A separable topological space is called a Hausdorff space, and has a distinct limit point. A topological monoid is an algebraic structure isomorphic to endomorphism if it is separable (i.e., Hausdorff) and comparable to an ultrametric [2]. However, the restriction of such endomorphism is that its left coset needs to be non-expansive in nature under composition. The permutation groups and monoid transformations follow topological structure and have point-wise convergence. A topological group is topologically isomorphic to the group automorphism and has a countable structure. In such cases, the topological group is a Polish group and non-Archimedean, and has a base at its identity element [2,3]. A group is called Abelian if the composition is commutative, generating normal subgroups in every case [4]. However, not all Abelian groups inherit any endomorphic ring within compact ring topology [4,5]. Moreover, an Abelian group can be constructed considering open J-radicals (Jacobson radicals) in a ring topology, which is finite [5]. The normed ring structures are the generalization of commutative rings. The fuzzy normed ring is constructed based on continuous $t$-norm and s-norm based on a fuzzy set [6]. Interestingly, the ideals are present within the fuzzy normed ring structure. A set of orientation-preserving topological actions on finite and non-Abelian groups is proposed [7]. The construction requires the group be finite and compact. In the algebra of multivalued group or semigroup, the EL-hypergroups are constructed having cyclic structure [8,9]. 
Compact fuzzy topological spaces are proposed, and the respective measures on L-fuzzy pretopological spaces are constructed [10-13]. The fuzzy topological spaces are modified by integrating multiple functors maintaining fuzzy compactness [12]. The topological fuzzy measure in distributed monoid spaces is formulated under topological local isomorphism and local homeomorphism [14]. It is considered that the underlying space is Hausdorff in nature. The fuzzy regular measure on Borel-set-based topological spaces is proposed preserving monotone class [15]. However, the regular fuzzy measure may not be strictly finite. In general, the maintenance of the uniform continuity of functions in a function space is difficult under fuzzification.

The definition of continuity of fuzzified functions is proposed considering Hausdorff metric space [16]. The compact-support-based fuzzy ultrametric was introduced, considering probability measures sets, in [17]. On the other hand, measures of noncompact fuzzy subsets in standard as well as fuzzy metric spaces were formulated in [18]. The algebraic and topological properties of fuzzy subsets were proposed considering related alpha-level subsets in [19]. The endomorphism of a fully disconnected group was formulated considering local compactness, where the scaling function was not always continuous with respect to Braconnier automorphic topology in [20].

This paper presents a detailed analysis of topological endomorphism and associated topological fuzzy measures in distributed monoid spaces. In general, it is considered that the space is Hausdorff and countable in nature. The monoids are cyclic type having distinct generators, and the related topological cyclic endomorphism within the subspace is formulated. The topology of the distributed monoid space is considered to be compact and second countable. The properties of topological fuzzy measures under cyclic topological endomorphism are presented.

The rest of the paper is organized as follows. Section 2 presents a set of preliminary concepts. Section 3 presents newly constructed definitions related to the presented model. Section 4 presents a set of analytical properties of the topological fuzzy measure under cyclic generators and endomorphism. Section 5 presents detailed comparative evaluations of related algebraic structures. Finally, Section 6 concludes the paper.

\section{Preliminaries}

Let $G=(X, *)$ be an algebraic group structure in 1D under the group operation given by $*: X^{2} \rightarrow X$, which is closed in $X$ [4]. The corresponding topology in 1D space is denoted as $\tau_{x} \subseteq P(X)$, maintaining the axioms of topological space [1]. Let $n \in Z^{+}, n>1$ be such that $A^{n} \subset X^{n}$ is equipped with a closed group operation in $n$-dimensional subspace as $o_{x}: A^{2 n} \rightarrow A^{n}$, generating a $G_{n}=\left(A^{n}, o_{x}\right)$ groupoid subspace [14]. Let $I \subset Z^{+}$be an index set. In $G_{n}=\left(A^{n}, o_{x}\right), M$ is a distributed monoid (DM) if the following properties are satisfied [14]:

$$
\begin{aligned}
& M=\left\{B_{i} \subset A^{n}: i \in I \subset Z^{+}\right\}, \\
& \cup B_{i}=A^{n}, \\
& B_{i} \cap B_{k}=\phi, \\
& \forall a_{i} \in B_{i}, \exists e_{i} \in B_{i}: a_{i} o_{x} e_{i}=e_{i} o_{x} a_{i}=a_{i}
\end{aligned}
$$

The corresponding structure $\left(X^{n}, M, \tau\right)$ is called a topological DM space if the following topological properties are satisfied in topology $\tau$ generated in $X^{n}$ :

$$
\begin{aligned}
& \tau \subseteq P\left(X^{n}\right), M \subset \tau, \\
& \tau \backslash M \neq \phi
\end{aligned}
$$

In topological DM space $\left(X^{n}, M, \tau\right)$, if $g: X^{n} \rightarrow R$ is a real-valued translation function such that $\forall a \in X^{n}, g(a) \in(-\infty, 0) \cup(0,+\infty)$, then $g\left(X^{n}\right)$ is a finite topological translation. A fuzzy topological measure in $\left(X^{n}, M, \tau\right)$ is defined as $\mu_{\tau}: \tau \rightarrow[0,1]$ such that [14] 


$$
\begin{aligned}
& \mu_{\tau}(\phi)=0, \exists \beta_{i} \in(1,+\infty), \\
& \forall D_{i} \in \tau, \mu_{\tau}\left(D_{i}\right)=\frac{1}{\beta_{i}} \sum_{\forall a \in D_{i}}\left|g(a)^{-1}\right|
\end{aligned}
$$

A fuzzy topological measure in topological DM space is measure consistent if $\mu_{\tau}\left(D_{i} \in \tau \backslash\left\{X^{n}\right\}\right)<1$. The bijection $f: M \rightarrow M$ in $\left(X^{n}, M, \tau\right)$ is a local isomorphism if $\forall B_{i} \in M, \exists B_{k} \in M: B_{i}=f^{-1}\left(B_{k}\right)$.

\section{Definitions}

In this section, a set of definitions extending the structure of topological DM space is introduced considering cyclic monoid generators and related endomorphism. It is considered that underlying topological DM space is Hausdorff and countable in nature.

\subsection{Generator Order}

In $\left(X^{n}, M, \tau\right)$ topological DM space, the order of $a_{i} \in B_{i} \in M$ is $O\left(a_{i}\right)=m$ if the following property is satisfied, where $\left(o_{x}\right)^{m}$ represents $m$-successive operations in a monoid:

$$
\begin{aligned}
& m \in Z^{+}, \\
& \forall a_{i} \in B_{i}, \exists m: a_{i}\left(o_{x}\right)^{m} a_{i}=e_{i} \in B_{i}, \\
& e_{i} o_{x} e_{i}=e_{i}
\end{aligned}
$$

If an element has $m=+\infty$, then it has infinite order [4]. However, in this paper it is considered that $m<+\infty$ in every monoid.

\subsection{Endomorphic Topological Map}

Let $\left(X^{n}, M, \tau\right)$ be a topological DM space. The surjective $v: X^{n} \rightarrow X^{n}$ is called endomorphic if the following axioms are maintained:

$$
\begin{aligned}
& D=\tau \backslash\{\phi\}, \\
& v(A \in D) \in \tau \backslash\{\phi, A\}, \\
& \forall B_{i} \in M, \exists A \in \tau \backslash\{\phi, M\}:\left(f_{\circ} v\right) A=f\left(B_{i}\right)
\end{aligned}
$$

The topological endomorphism preserves local symmetries within the underlying subspace.

\subsection{Cyclic Map}

In $\left(X^{n}, M, \tau\right)$ topological DM space, $\gamma:\left(B_{i} \in M\right) \rightarrow B_{i}$ is a cyclic map iff it maintains the following axioms:

$$
\begin{aligned}
& \forall a_{k} \in B_{i}, \exists k \in Z_{0}^{+}: \\
& a_{i}\left(o_{x}\right)^{k} a_{i}=a_{k} \\
& \gamma^{k}\left(a_{i}\right)=a_{k}
\end{aligned}
$$

It is considered that $a_{i} \in B_{i}$ is a cyclic generator of the corresponding monoid in the topological subspace.

\subsection{Cyclic Topological Endomorphism}

Let $\left(X^{n}, M, \tau\right)$ be a topological DM space. A cyclic topological endomorphism in $\left(X^{n}, M, \tau\right)$ is given by $u: X^{n} \rightarrow X^{n}$ iff it maintains the following axioms:

$$
\begin{aligned}
& \forall B_{i} \in M, \exists A \in \tau \backslash M: u(A)=a_{i} \in B_{i}, \\
& A \neq \phi,|A|<\left|X^{n}\right|, m \in[0,+\infty), \\
& \bigcup_{t=0}^{m}\left\{\left(\gamma^{t} \circ u\right) A\right\}=B_{i}
\end{aligned}
$$


The cyclic topological endomorphism generates a set of finite distributed monoid subspaces in $\left(X^{n}, M, \tau\right)$.

\section{Analytical Properties}

Theorem 1. In the $\left(X^{n}, M, \tau\right)$ Hausdorff and countable topological DM space, $\mu_{\tau}(D \in \tau \backslash\{\phi\})>0$.

Proof: Let $\left(X^{n}, M, \tau\right)$ be a Hausdorff countable topological DM space and $\tau=P\left(X^{n}\right)$. Thus, $\forall D \in \tau \backslash\{\phi\}$ in $\left(X^{n}, M, \tau\right)$, it is true that given a set of natural numbers $N, \exists \omega($.$) such that \omega: N \rightarrow D$. It follows that $[D \neq \phi] \Rightarrow[n \geq 1]$, where $\omega(n \in N) \subseteq D$. Moreover, $\forall a \in D, g(a) \neq 0$, indicating that $\mu_{\tau}(D \neq \phi)>0$, where $\left|g(a)^{-1}\right| \in(0,+\infty)$.

Theorem 2. If the $\left(X^{n}, M, \tau\right)$ topological DM space is Hausdorff, then $\mu_{\tau}\left(X^{n} \backslash \cup_{i \in I}\left(D_{i} \in \tau\right)\right)=0$, where $D_{i}=\underset{k \in I}{\cup} B\left(\varepsilon>0, a_{k} \in X^{n}\right)$.

Proof: Let $\left(X^{n}, M, \tau\right)$ be a Hausdorff topological DM space. If $X^{n}$ is compact, then $\partial X^{n} \subset \overline{X^{n}}$. Let $D_{i}=\underset{k \in I}{\cup} B\left(\varepsilon>0, a_{k} \in X^{n}\right)$ be a set of open balls such that $B\left(\varepsilon>0, a_{k} \in X^{n}\right) \cap B\left(\varepsilon>0, a_{i} \in X^{n}\right)=\phi$ if $a_{k} \neq a_{i}$, maintaining Hausdorff property. This indicates that $\overline{X^{n}} \backslash \cup \cup\left(D_{i} \in \tau \backslash\{\phi\}\right)=\partial X^{n} \cup\{\phi\}$, which is a Baire category set (nowhere dense). Hence, $X^{n} \backslash \bigcup_{i \in I}\left(D_{i} \in \tau\right)=\phi$, resulting in $\mu_{\tau}\left(X^{n} \backslash \bigcup_{i \in I}\left(D_{i} \in \tau\right)\right)=0$.

Theorem 3. In the $\left(X^{n}, M, \tau\right)$ topological DM space, $\mu_{\tau}$ is measure consistent if $\beta_{i} \geq \sum_{\forall a \in D_{i} \in \tau \backslash\langle\phi\}}\left|g(a)^{-1}\right|$.

Proof: Let $\left(X^{n}, M, \tau\right)$ be a Hausdorff topological DM space equipped with finite translation, $\left|g\left(a \in X^{n}\right)^{-1}\right| \in(0,+\infty)$. However, $\forall D_{i} \in \tau \backslash\left\{\phi, X^{n}\right\}$ in the topological space and $\exists \beta_{i} \in(0,+\infty)$ such that $\left[\left(0<\mu_{\tau}\left(D_{i}\right) \leq 1\right) \wedge\left|D_{i}\right|=1\right] \Rightarrow\left[\left|g\left(D_{i}\right)^{-1}\right| \leq \beta_{i}\right]$. Inductively, if $n=\left|D_{i}\right|$ and $m=\sum_{\forall a \in D_{i}}\left|g(a)^{-1}\right|$, then $\left[\left(0<\mu_{\tau}\left(D_{i}\right) \leq 1\right) \wedge n>1\right] \Rightarrow\left[m \leq \beta_{i}+(\varepsilon>0)\right]$. Furthermore, as the underlying space is Hausdorff, it is thus true that $\left[\left|D_{i} \in \tau\right|=1\right] \Rightarrow\left[\mu_{\tau}\left(D_{i} \backslash B\left(\varepsilon>0, a \in D_{i}\right)\right)=0\right]$. Hence, it is measure consistent in $\left(X^{n}, M, \tau\right)$.

Theorem 4. If $\left(X^{n}, M, \tau\right)$ is a countable Hausdorff topological DM space, then $\mu_{\tau}\left(D_{i} \in \tau \backslash\{\phi\}\right) \leq$ $\sum_{i \in I} \mu_{\tau}\left(B\left(\varepsilon>0, a_{i} \in D_{i}\right)\right)$, depending on $\varepsilon$.

Proof: Let $\left(X^{n}, M, \tau\right)$ be a countable topological DM space, and $D_{i} \in \tau \backslash\{\phi\}$. Thus, $\forall B\left(\varepsilon>0, a_{i} \in D_{i}\right) \subset D_{i}$, the following condition is valid, $\mu_{\tau}\left(B\left(\varepsilon>0, a_{i} \in D_{i}\right)\right) \in(0,1)$. Moreover, as the space is Hausdorff, $\forall a_{i}, a_{k} \in X^{n}, B\left(\varepsilon>0, a_{i}\right) \cap B\left(\varepsilon>0, a_{k}\right)=\phi$. Let $\delta>\varepsilon$ be such that $\forall a_{i}, a_{k} \in D_{i}, B\left(\delta, a_{i}\right) \cap B\left(\delta, a_{k}\right) \neq \phi$ and $\cup_{i \in I} B\left(\delta, a_{i}\right) \subseteq D_{i}$. Thus, it is true that $\mu_{\tau}\left(B\left(\delta, a_{i}\right) \cap B\left(\delta, a_{k}\right)\right)>0$ in the corresponding measure space. Hence, $\mu_{\tau}\left(D_{i} \in \tau \backslash\{\phi\}\right) \leq \sum_{i \in I} \mu_{\tau}\left(B\left(\delta>0, a_{i} \in D_{i}\right)\right)$ in the underlying $\left(X^{n}, M, \tau\right)$, depending on $\delta \geq \varepsilon$.

Theorem 5. In topologically endomorphic $\left(X^{n}, M, \tau\right)$, if $\forall a \in X^{n}, g(a)=k \in R^{+}$, then $\mu_{\tau}\left(A \in \tau \backslash\left\{\phi, X^{n}\right\}\right) \geq$ $\mu_{\tau}\left(\left(f_{0} v\right) A\right)$ under uniform $\beta$.

Proof: Let $\left(X^{n}, M, \tau\right)$ be a topological endomorphic DM space equipped with measure under uniform $\beta$. Let $A \subset X^{n}$ be such that $A \in \tau \backslash\left\{\phi, X^{n}\right\}$, and $|v(A)| \leq|A|$. If $\exists B_{i} \in M$ such that $\left(f_{\circ} v\right) A=f\left(B_{i}\right)$, then 
$\left|f\left(B_{i}\right)\right| \leq|A|$ due to bijective local isomorphism. Moreover, as $\forall a \in X^{n}, g(a)=k \in R^{+}, \sum_{i \in I}\left|g\left(a_{i} \in A\right)^{-1}\right| \geq$ $\sum_{i \in I}\left|g\left(a_{i} \in\left(f_{\circ} v\right) A\right)^{-1}\right|$.

Hence, $\mu_{\tau}\left(A \in \tau \backslash\left\{\phi, X^{n}\right\}\right) \geq \mu_{\tau}\left(\left(f_{\circ} v\right) A\right)$ under uniform $\beta$ in the space.

Theorem 6. In topologically endomorphic $\left(X^{n}, M, \tau\right)$, if $\mu_{\tau}\left(A \subset X^{n}\right)=\mu_{\tau}\left(\left(f_{\circ} v\right) A\right)$, then $\forall a_{i}, a_{k} \in A, g\left(a_{i}\right) \neq g\left(a_{k}\right)$.

Proof: Let $\left(X^{n}, M, \tau\right)$ be a topological endomorphic DM space with uniform translation in $A$, and $\mu_{\tau}\left(A \subset X^{n}\right)=\mu_{\tau}\left(\left(f_{\circ} v\right) A\right)$. Let $D \subset A$ such that $\mu_{\tau}(D)=\mu_{\tau}\left(\left(f_{\circ} v\right) D\right)$, where $|D|=\left|\left(f_{\circ} v\right) D\right|$. This indicates that $\mu_{\tau}(A)=\mu_{\tau}(D)+(\delta>0)$, and $\delta=\mu_{\tau}\left(\left(f_{\circ} v\right)(A \backslash D)\right)$. However, if $|A|>\left|f\left(B_{i} \in M\right)\right|$, then $\mu_{\tau}(A \backslash D) \neq \delta$, which is a contradiction. Hence, translation is not uniform in $A$, indicating $\forall a_{i}, a_{k} \in A, g\left(a_{i}\right) \neq g\left(a_{k}\right)$.

Lemma 1. In topologically endomorphic $\left(X^{n}, M, \tau\right)$, if $\forall a_{i}, a_{k} \in X^{n}, g\left(a_{i}\right)=g\left(a_{k}\right)$, then $\mu_{\tau}\left(A \in \tau \backslash\left\{\phi, X^{n}\right\}\right)=$ $\mu_{\tau}\left(\left(f_{\circ} v\right) A\right)+(c>0)$.

Proof: Let in $\left(X^{n}, M, \tau\right)$ be $\exists A \subset X^{n},|A|>\left|B_{i} \in M\right|$, and $\left(f_{\circ} v\right) A=f\left(B_{i}\right)$ within the endomorphic space. If $\forall a, b \in X^{n}, g(a)-g(b)=0$, and $D \subset A: \mu_{\tau}(D)=\mu_{\tau}\left(\left(f_{0} v\right) D\right)$, then $\mu_{\tau}(D)=\mu_{\tau}\left(f\left(B_{i}\right)\right)$ within the space. This indicates that $\mu_{\tau}(A \backslash D)>0$. Hence, $\mu_{\tau}\left(A \in \tau \backslash\left\{\phi, X^{n}\right\}\right)=\mu_{\tau}\left(\left(f_{\circ} v\right) A\right)+(c>0)$ in $\left(X^{n}, M, \tau\right)$.

Theorem 7. In the cyclic topological endomorphic space $\left(X^{n}, M, \tau\right), \mu_{\tau}\left(B_{i} \in M\right)=\sum_{m=0}^{L} \mu_{\tau}\left(\left(\gamma^{m} \circ u\right) A\right)$.

Proof: Let $N$ be a set of natural numbers and $B_{i} \in M$ in $\left(X^{n}, M, \tau\right)$ have cyclic endomorphism such that $\chi: N \rightarrow B_{i}$ and $\chi(n)=a_{n}$, where $\chi(n) \neq \chi(m)$. If $A \in \tau \backslash\left\{\phi, M, X^{n}\right\}$ exists in the space such that $u(A)=\chi(i) \in B_{i}$, then $a_{k}=\left(\gamma^{k}{ }_{\circ} u\right) A$. However, $B_{i}=\bigcup_{n=1}^{K}\{\chi(n)\}$, where $K=\left|B_{i}\right|$ and $\mu_{\tau}\left(B_{i}\right)=\sum_{n=1}^{K} \mu_{\tau}(\{\chi(n)\})$. Hence, $\mu_{\tau}\left(B_{i} \in M\right)=\sum_{m=0}^{L} \mu_{\tau}\left(\left(\gamma^{m}{ }_{\circ} u\right) A\right)$ for some $L \in N$ in cyclic endomorphic $\left(X^{n}, M, \tau\right)$ topological DM space.

Theorem 8. In cyclic endomorphic $\left(X^{n}, M, \tau\right)$ space, $\exists \lambda>0$ such that $\mu_{\tau}\left(A \subset X^{n}\right)=\lambda \mu_{\tau}\left(\bigcup_{m=0}^{K}\left\{\left(\gamma^{m}{ }_{\circ} u\right) A\right\}\right)$.

Proof: Let $\left(X^{n}, M, \tau\right)$ be a cyclic endomorphic topological DM space. Let $\exists A \subset X^{n}, \exists B_{i} \in M$ such that $u(A)=a_{i} \in B_{i}$ and $\bigcup_{m=0}^{K}\left\{\left(\gamma^{m} \circ u\right) A\right\}=B_{i}$. This indicates that $A \in \tau \backslash\left\{\phi, M, X^{n}\right\}$ in the space. Now, if $\forall a, b \in X^{n}, g(a)=g(b)$, then $\exists \beta \in(1,+\infty), \lambda=1$ such that $\mu_{\tau}(A)=\mu_{\tau}\left(\bigcup_{m=0}^{K}\left\{\left(\gamma^{m} \circ u\right) A\right\}\right)$. However, if $\forall a, b \in X^{n}, g(a) \neq g(b)$, then $\exists \lambda \in(0,+\infty) \backslash\{1\}$ such that $\mu_{\tau}(A)=\lambda \mu_{\tau}\left(\bigcup_{m=0}^{K}\left\{\left(\gamma^{m}{ }_{\circ} u\right) A\right\}\right)$. Hence, in any case $\exists \lambda>0$ such that $\mu_{\tau}(A)=\lambda \mu_{\tau}\left(\bigcup_{m=0}^{K}\left\{\left(\gamma^{m} \circ u\right) A\right\}\right)$, where $A \in \tau \backslash\left\{\phi, M, X^{n}\right\}$.

Corollary 1. If $\mu_{\tau}(A)=\lambda \mu_{\tau}\left(\bigcup_{m=0}^{K}\left\{\left(\gamma^{m} \circ u\right) A\right\}\right)$, then $\exists \lambda_{c}>0$ such that $\mu_{\tau}(A)=\lambda_{c} \mu_{\tau}(u(A))$, where $\lambda_{c} \neq \lambda$.

Theorem 9. In topologically cyclic endomorphic $\left(X^{n}, M, \tau\right)$ space, $\mu_{\tau}\left(\bigcup_{m=0}^{r}\left\{\left(\gamma^{m} \circ u\right) A\right\}\right)<\mu_{\tau}\left(\bigcup_{m=0}^{s}\left\{\left(\gamma^{m} \circ u\right) A\right\}\right)$ if $r<s<K$.

Proof: Let $N$ be a set of natural numbers and $\left(X^{n}, M, \tau\right)$ be a topologically cyclic endomorphic space with DM embeddings. In the underlying subspace $M \subset \tau$ and $\forall B_{i} \in M, \mu_{\tau}\left(B_{i}\right) \in(0,1)$. However, 
if $A \in \tau \backslash\left\{\phi, M, X^{n}\right\}$ such that $u(A)=a_{i} \in B_{i}$, then $\exists K \in N$, maintaining $\bigcup_{m=0}^{K}\left\{\left(\gamma^{m} \circ u\right) A\right\}=B_{i}$. Thus, $\exists r, s \in N, r<s<K$ such that $\left(\bigcup_{m=0}^{r}\left\{\left(\gamma^{m} \circ u\right) A\right\}\right) \subset\left(\bigcup_{m=0}^{s}\left\{\left(\gamma^{m} \circ u\right) A\right\}\right)$.

$$
\text { Hence, } \mu_{\tau}\left(\bigcup_{m=0}^{r}\left\{\left(\gamma^{m} \circ u\right) A\right\}\right)<\mu_{\tau}\left(\bigcup_{m=0}^{s}\left\{\left(\gamma^{m} \circ u\right) A\right\}\right) \text { as } \forall a \in X^{n}, g(a) \neq 0 \text {. } \square
$$

Theorem 10. In cyclic endomorphic topological DM space, if order $O\left(a_{k} \in B_{i}\right)=n$, then $\mu_{\tau}\left(\left(a_{k}\left(o_{x}\right)^{n} a_{k}\right) o_{x} E\right)=$ $\mu_{\tau}(E)$, where $E=\bigcup_{m=0}^{r<K}\left\{\left(\gamma^{m} \circ u\right) A\right\}$ and, $\mu_{\tau}(E)<\mu_{\tau}\left(B_{i}\right)$.

Proof: Let $\left(X^{n}, M, \tau\right)$ be a cyclic endomorphic topological DM space. Let $A \in \tau \backslash\left\{\phi, M, X^{n}\right\}$ in the underlying space such that $\left(B_{i} \in M\right)=\bigcup_{m=0}^{K}\left\{\left(\gamma^{m}{ }_{\circ} u\right) A\right\}$. Thus, $\exists E \subset B_{i}$, where $E=\bigcup_{m=0}^{r<K}\left\{\left(\gamma^{m}{ }_{\circ} u\right) A\right\}$ and $\mu_{\tau}(E) \in(0,1)$. However, if $O\left(a_{k} \in B_{i}\right)=n$, then $\left(a_{k}\left(o_{x}\right)^{n} a_{k}\right) o_{x} E=E$ within DM space. Thus, $\mu_{\tau}\left(\left(a_{k}\left(o_{x}\right)^{n} a_{k}\right) o_{x} E\right)=\mu_{\tau}(E)$ and $\mu_{\tau}(E)<\mu_{\tau}\left(B_{i}\right)$ in $\left(X^{n}, M, \tau\right)$.

\section{Comparative Evaluation}

Measurable spaces can be constructed considering various types of embedded algebraic structures, as well as associated translation functional properties within the spaces. In this section, comparisons of the proposed fuzzy measure in cyclic endomorphic topological monoid spaces are presented by considering the measure-invariant properties of topological groups, various translations, the probabilistic nature of group measure as well as the endomorphic measure of groups and semigroups. Accordingly, the respective algebraic structures are first classified, and the properties of the respective measures under translations are presented. The comparative evaluation of the proposed fuzzy topological measure in cyclic endomorphic DM space (denoted as EFM) is presented considering four different categories of topological and group model, namely, topological actions on groups (TAG) [7], measure-invariant semigroups (MIS) [21], probability-measured Lie groups (PML) [22], and endomorphic measure on groups (EMG) [23]. First, the models are categorized based on different classes of group structures and their associated topological properties as illustrated in Table 1.

Table 1. Comparison of models based on group structures and topologies. EFM: fuzzy topological measure in cyclic endomorphic distributed monoid (DM) space; EMG: endomorphic measure on groups; MIS: measure-invariant semigroups; PML: probability-measured Lie groups; TAG: topological actions on groups.

\begin{tabular}{ccccc}
\hline $\begin{array}{c}\text { Group and Topological } \\
\text { Properties }\end{array}$ & Compactness/Finiteness & Commutativity & Topology & Embeddings \\
\hline Model: TAG & Finite & Non-Abelian & Orientation-preserving & Fixed point \\
Model: MIS & Locally compact & Abelian & Translation-invariant & Not applicable \\
Model: PML & Borel sets & Not applicable & Weak convergent & Not applicable \\
Model: EMG & Amenable locally compact & Polish groups & Second-countable & Not applicable \\
Model: EFM & Finite, compact & Not applicable & Second-countable & Monoids \\
\hline
\end{tabular}

The TAG model considers finite and non-Abelian groups, where the topological actions are orientation preserving in nature. The topological orientation-preserving TAG model embeds fixed points in topological spaces, maintaining isomorphism. However, the MIS model considers that the underlying group structure is Abelian and locally compact. The PML model of measure is based on Lie groups in weak-convergent topological spaces. On the other hand, the PML model is based on Polish groups, which are amenable and locally compact within the space. In this respect, the proposed EFM formulation is distinct, because the embedded monoids are finite, disjoint, and compact. Moreover, the distributed monoids of EFM are cyclic and have embeddings within the endomorphic topological space. The EFM model proposed in this paper preserves the consistency of fuzzy measures irrespective 
of the commutativity of monoids. Moreover, the underlying topological space is considered to be Hausdorff and second countable. The corresponding comparative evaluation of the properties of translation functions and associated measures are presented in Table 2.

Table 2. Comparison of translation and measure properties.

\begin{tabular}{|c|c|c|c|c|c|c|}
\hline $\begin{array}{l}\text { Measure } \\
\text { Properties }\end{array}$ & Translation & Symmetry & $\begin{array}{l}\text { Isomorphism/ } \\
\text { Endomorphism }\end{array}$ & Finiteness & $\begin{array}{c}\text { Haar } \\
\text { Condition }\end{array}$ & Measure \\
\hline Model: TAG & Orientation-preserving & No & Isomorphic & Not applicable & Not applicable & Not applicable \\
\hline Model: MIS & Linear additive & No & Translation-invariant & Finite & Not applicable & Non-fuzzy \\
\hline Model: EMG & Measure-preserving & Not applicable & Automorphic & Sigma-finite & Yes & Probability-preserving \\
\hline Model: EFM & Linear or non-linear & $\begin{array}{l}\text { Depends on } \\
\text { translation }\end{array}$ & Endomorphic & Yes & Yes & Fuzzy measure \\
\hline
\end{tabular}

In the MIS group, the measure is invariant to translation within the space, where the translation function is linear additive. The MIS measure is in a non-Fuzzy class without any inherent symmetry. However, in case of the compact Lie groups (PML), the measure is dependent on respective Borel sets. The PML measure is a symmetric measure preserving Haar measurability conditions. In the measure of Polish-group class (EMG), the translation function is measure preserving and automorphic in nature. The corresponding measure is a sigma-finite probability measure and is Haar measurable. However, the proposed EFM model considers finite fuzzy measures, which preserves the least Haar measurability based on cyclic monoids. The distinctive feature of the proposed EFM formulation is that the cyclic distributed monoids containing distinct generators are embedded in endomorphic topological space. The topological endomorphism of the space preserves the consistency of fuzzy measure based on an appropriate finite real-valued translation function. The underlying space of EFM is topologically endomorphic, and the symmetry of the measure is dependent on the applied translation functions within the space. The non-uniformity of translation and the uniformity of the scaling factor preserve the symmetry and consistency of fuzzy measures in endomorphic topological space. The cyclic monoids include torsion elements having finite order in the proposed EFM analytical formulation while preserving the consistency of finite fuzzy measures. Finally, the proposed analytical construction maintains the monotonicity and countable additivity properties of fuzzy measures in the presence of cyclic endomorphism.

\section{Conclusions}

Fuzzy set theory has applications in systems with inherent uncertainties, and the corresponding fuzzy measure theory has numerous computational applications. The properties of fuzzy measures in topological spaces are interesting from the analytical point of view in the presence of embedded distributed monoids in the spaces. The existence of cyclic topological endomorphism provides a new perspective about topological fuzzy measures, as presented in a set of theorems. The cyclic generators in topologically distributed monoid spaces influence the corresponding fuzzy measures, where the space is Hausdorff and countable in nature. Furthermore, the cyclic endomorphic monoid subspaces are considered to be finite and compact, without any restriction on commutativity. The proposed fuzzy measure successfully preserves finiteness in endomorphic topological space having distributed monoid embeddings. However, unlike probabilistically measured Lie groups, the symmetry of the proposed fuzzy measure is dependent on the nature of the translation function. The translation function can be linear or non-linear in nature, preserving the measure within subspaces. Unlike the locally compact disconnected group having discontinuous scaling with respect to automorphic Braconnier topology, the proposed endomorphic fuzzy measure considers the existence of cyclic locally compact distributed monoids. The comparative studies presented in this paper explain the distinguishing features of the proposed topological fuzzy measure in the presence of cyclic endomorphism.

Author Contributions: Susmit Bagchi is sole author of this paper and contributed in full. 
Funding: The funding of this work is partly covered by Gyeongsang National University, Jinju, ROK.

Acknowledgments: The author would like to thank the editors and anonymous reviewers for their helpful comments and suggestions.

Conflicts of Interest: The author declares no conflict of interest.

\section{References}

1. Bourbaki, N. General Topology; Springer: Berlin, Germany, 1998; Volume 1.

2. Bodirsky, M.; Schneider, F.M. A topological characterization of endomorphism monoids of countable structures. Algebra Univers. 2017, 77, 251-269. [CrossRef]

3. Becker, H.; Kechris, A. The Descriptive Set Theory of Polish Group Actions; LMS Lecture Note Series, No. 232; Cambridge University Press: Cambridge, UK, 1996.

4. Rudin, W. Measure algebras on abelian groups. Bull. Am. Math. Soc. 1959, 65, 227-247. [CrossRef]

5. Ursul, M.; Juras, M. Topological rings of endomorphisms. Commun. Algebra 2010, 38, 1421-1435. [CrossRef]

6. Emniyet, A.; Sahin, M. Fuzzy normed rings. Symmetry 2018, 10, 515. [CrossRef]

7. Zimmermann, B.P. On topological actions of finite groups on $S^{3}$. Topol. Appl. 2018, 236, 59-63. [CrossRef]

8. Novak, M.; Krehlik, S.; Cristea, I. Cyclicity in EL-Hypergroups. Symmetry 2018, 10, 611. [CrossRef]

9. Novák, M.; Křehlík, Š. EL-hyperstructures. Soft Comput. 2018, 22, 7269-7280. [CrossRef]

10. Shi, F.G.; Liang, C. Measures of compactness in L-fuzzy pretopological spaces. J. Intell. Fuzzy Syst. 2014, 26, 1557-1561.

11. Fang, J. Sums of L-fuzzy topological spaces. Fuzzy Sets Syst. 2005, 157, 739-754. [CrossRef]

12. Lowen, R. Fuzzy topological spaces and fuzzy compactness. J. Math. Anal. Appl. 1976, 56, 621-633. [CrossRef]

13. Chang, C.L. Fuzzy topological spaces. J. Math. Anal. Appl. 1968, 24, 182-190. [CrossRef]

14. Bagchi, S. On the analysis and computation of topological fuzzy measure in distributed monoid spaces. Symmetry 2019, 11, 9. [CrossRef]

15. Wu, J.; Wu, C. Fuzzy regular measures on topological spaces. Fuzzy Sets Syst. 2001, 119, 529-533. [CrossRef]

16. Wu, H.C. Continuity of fuzzyfied functions using the generalized extension principle. Symmetry 2017, 9, 299. [CrossRef]

17. Savchenko, A.; Zarichnyi, M. Fuzzy ultrametrics on the set of probability measures. Topology 2009, 48, 130-136. [CrossRef]

18. Ban, A.I.; Gal, S.G. Measures of noncompactness for fuzzy sets in fuzzy topological spaces. Fuzzy Sets Syst. 2000, 109, 205-216. [CrossRef]

19. Onasanya, B.O.; Hoskova-Mayerova, S. Some Topological and Algebraic Properties of alpha-level Subsets' topology of a fuzzy subset. An. Stiintifice Ale Univ. Ovidius Constanta Ser. Mat. 2018, 26, 213-227. [CrossRef]

20. Willis, G.W. Computing the scale of an endomorphism of a totally disconnected locally compact group. Axioms 2017, 6, 27. [CrossRef]

21. Rigelhof, R. Invariant measures on locally compact semigroups. Proc. Am. Math. Soc. 1971, 28, $173-176$. [CrossRef]

22. Applebaum, D. Probability on Compact Lie Groups, Probability Theory and Stochastic Modelling 70; Springer: Berlin, Germany, 2014; pp. 81-118.

23. Danilenko, A.I. Endomorphisms of measured equivalence relations, cocycles with values in non-locally compact groups and applications. Ergod. Theory Dyn. Syst. 1999, 19, 571-590. [CrossRef] 